\title{
INTEGRAL AND $p$-MODULAR SEMISIMPLE DEFORMATIONS FOR $p$-SOLVABLE GROUPS OF FINITE REPRESENTATION TYPE
}

\author{
MARY SCHAPS
}

(Received 4 April 1989)

Communicated by $\mathbf{H}$. Lausch

\begin{abstract}
We prove that the split integral group ring of a finite $p$-solvable group of finite representation type has a structure analogous to that of the $p$-modular semisimple deformation. The split integral deformation can be put in the same form as the $p$-modular deformation by an appropriate substitution for the parameter $T$. As an application we derive a simple formula for the matrix units in the semisimple group algebra over a nonmodular prime.
\end{abstract}

1980 Mathematics subject classification (Amer. Math. Soc.) (1985 Revision): 16 A 58, 20 C 05.

\section{Introduction}

In [2] Donald and Flanigan conjectured a modular version of Maschke's theorem, namely that for every finite group and every sufficiently large field $k$, the group algebra $k G$ has a deformation to a semisimple algebra with the same Wedderburn components as the group algebra over a field of characteristic 0 . We will call such a deformation a $p$-modular semisimple deformation. This conjecture is only of interest in the $p$-modular case, when the characteristic $p$ of $k$ divides $|G|$. In the nonmodular case, Maschke's theorem tells us that $k G$ is already semisimple and no deformation is needed.

Donald and Flanigan [2] proved the original conjecture for commutative groups twenty years ago. The author [10] just recently proved the conjecture for groups of finite representation type, that is, groups with cyclic $p$-Sylow subgroup.

(C) 1991 Australian Mathematical Society 0263-6115/91 \$A2.00+0.00 
The current paper addresses the motivation for conjecturing the existence of a $p$-modular semisimple deformation, and consequences of finding such a deformation, in one case of finite representation type for which the theory is particularly simple and elegant: the $p$-solvable groups with cyclic $p$ Sylow subgroup. One of the primary reasons for conjecturing the existence of a unicharacteristic $p$-modular semisimple deformation is that the split integral group ring provides a "multicharacteristic" semisimple deformation. We show in this paper that in the $p$-solvable case the two deformations are entirely analogous.

\section{Background and notation}

Let $|G|=m e$, with $m=p^{c}$ and $(e, p)=1$. A $p$-number is a power of $p$, and a $p^{\prime}$-number is a number relatively prime to $p$. Let $O_{p^{\prime}}(G)$ be the maximal normal $p^{\prime}$-subgroup of $G$. Let $P=S_{p}(G)$ be a $p$-Sylow subgroup of $G$, and let $Z(G)$ be the center of $G$. Let $G^{\prime}$ be the commutator subgroup and let $C_{n}$ be the cyclic group of order $n$.

Let $R$ be a commutative ring, and $R^{*}$ its group of units. A Hochschild cocycle $\alpha$ is a function $\alpha: G \times G \rightarrow R^{*}$ satisfying the identities

$$
\begin{gathered}
\alpha(x, 1)=\alpha(1, x)=1, \\
\alpha(x, y) \alpha(x y, z)=\alpha(y, z) \alpha(x, y z) \text { for } x, y, z \in G .
\end{gathered}
$$

We denote the set of cocycles by $Z^{2}\left(G, R^{*}\right)$. The coboundaries $B^{2}\left(G, R^{*}\right)$ are the cocycles determined by a map $\beta: G \rightarrow R^{*}$ according to the formula

$$
\alpha(x, y)=\beta(x) \beta(y)(\beta(x y))^{-1} .
$$

Define $H^{2}\left(G, R^{*}\right)=Z^{2}\left(G, R^{*}\right) / B^{2}\left(G, R^{*}\right)$. We denote by $R G$ the standard group algebra with the elements of the group as basis and multiplication determined by the group multiplication. We denote by $R^{\alpha} G$ the twisted group algebra on the same basis with multiplication induced by $g \cdot h=$ $\alpha(g, h) g h$.

Definition. A group is $p$-solvable if it has a composition series $G \triangleright G_{1} \triangleright$ $G_{2} \triangleright \cdots \triangleright 0$ in which every factor group is either a $p$-group or a $p^{\prime}$-group.

Definition. A $k$-algebra deformation $A$ of an $n$ dimensional $k$-algebra $A_{0}$ over a commutative $k$-algebra $R$ is an associative unitary multiplication structure, on a basis $x_{1}, \ldots, x_{n}$ of the form

$$
x_{i} \cdot x_{j}=\sum_{q=1}^{n} a_{i j}^{q} x_{q} \text {, }
$$


with structure constants $a_{i j}^{q} \in R$ such that the residues of the $a_{i j}^{q}$ modulo some maximal ideal $m_{0}$ of $R$ give structure constants for the algebra $A_{0}$.

In the current case it suffices to take $R=k[T]$ for an indeterminate $T$, and let the special point be given by setting $T=0$. If all the algebras $A_{s}$ obtained by setting $T=s$ for $s \neq 0$ are isomorphic to $A_{1}$, then we also say that $A_{1}$ is a deformation of $A_{0}$.

EXAMPLE 1. If $m=p^{c}$, with $\operatorname{char} k=p$, then let $G$ be the cyclic group of order $m$, and take $A_{0}$ to be the group algebra $k G$, which is isomorphic to $k[z] /(z)^{m}$. Define a deformation $A$ of $A_{0}$ to be the $k[T]$-algebra with basis $1, z, z^{2}, \ldots, z^{m-1}$ and multiplication

$$
z^{i} z^{j}= \begin{cases}z^{i+j} & \text { if } i+j<m, \\ T^{m-1} z^{i+j-m+1} & \text { if } i+j \geq m .\end{cases}
$$

For $T=0$ this is $A_{0}$ and for all nonzero values $s$ of $T$ it is the semisimple commutative algebra $k^{m} \stackrel{\sim}{\rightarrow} k[Z] /\left(Z^{m}-s^{m-1} Z\right)$.

Deinition. A multicharacteristic deformation $A$ of a $k$-algebra $A_{0}$ over a parameter ring $R$ is a multiplication structure as above, except that $R$ is a multicharacteristic ring.

Definition. Let $K$ be a finite extension of the rational numbers containing all roots of unity of order at most $|G|$. Let $O$ be the ring of integers in $K$, that is, the integral closure of $\mathbb{Z}$ in $K$. Let $S$ be the set of all primes dividing $|G|$ which don't divide $p$, and let $O_{S}$ be the subring of $K$ containing $O$ in which elements of $S$ are inverted. The group ring $O_{S}[G]$ will be called the split integral group ring.

REMARK. Over every prime not dividing $|G|$ it splits into a semisimple group algebra, since $O_{S}$ will contain the necessary roots of unity all of which are integral over $\mathbb{Z}$.

\section{The $p$-modular semisimple deformation for $p-p^{\prime}$ metacyclic groups}

We now construct the $p$-modular semisimple deformation for one important special case.

Definition. A $p-p^{\prime}$ metacyclic group is a group with a cyclic normal $p$ Sylow subgroup and a cyclic quotient.

We fix a presentation for such a group. Let $a$ be a generator for a $p$ Sylow subgroup $P=S_{p}(G)$, of order $m=p^{c}$. Let $e=|G / P|$, so that $(e, p)=1$. Let $b$ be an element of $G$ which induces a generator of $G / P$. Since conjugation by $b$ is an automorphism of $P, b a b^{-1}=a^{r}$ with $a^{r}$ another generator of $P$, that is, with $(r, p)=1$. Furthermore, since $b^{e} \in P$, 
which is abelian, $a=b^{e} a b^{-e}=a^{r^{e}}$. Thus $r^{e} \equiv 1(\bmod m)$, and $G$ is nonabelian if $r \not \equiv 1(\bmod m)$. By the Schur-Zassenhaus Theorem $[1,8.35]$, $G$ is a semidirect product, so $b^{e}=1$. In summary we have a presentation

$$
G=\left\langle a, b \mid a^{m}=1, b^{e}=1, b a=a^{r} b, r^{e} \equiv 1(\bmod m)\right\rangle .
$$

We collect together the results which we will need about such groups. Since the proofs are mostly elementary number theory, we include them instead of referring the reader to general theorems.

LEMMA 1. Let $G$ be a nonabelian p-p' metacyclic group.

(1) $|Z(G)|$ is prime to $p$.

(2) If $Z(G)$ is trivial, then $e$ divides $p-1$ (and thus also $m-1$ ).

(3) If $Z(G)$ is trivial, then every subgroup $H$ of $G$ is a $p-p^{\prime}$ metacyclic group with trivial center, or is cyclic of $p$ or $p^{\prime}$ order.

(4) If $k$ is an algebraically closed field of characteristic not equal $p$, then $H^{2}\left(G, k^{*}\right)$ is trivial, i.e. every cocycle is a coboundary.

Proof. Recall that $|G|=m e$, with $m=p^{c}$.

(1) Suppose $a^{m^{\prime \prime}} \in Z(G)$, for $m^{\prime \prime}=p^{d}, d \leq c$. Then $a^{m^{\prime \prime}} b=b a^{m^{\prime \prime}}=$ $a^{m^{\prime \prime} r} b$, so $m^{\prime \prime}(r-1) \equiv 0(\bmod m)$. If $m \neq m^{\prime \prime}$, then $p \mid r-1$, so $r=u p^{c^{\prime}}+1$ with $(u, p)=1$. Since $G$ is nonabelian, $c^{\prime}<c$. However, since $1 \equiv r^{e}=$ $\left(u p^{c^{\prime}}+1\right)^{e}$, and $(e, p)=1$, we have $0 \equiv e \cdot u p^{c^{\prime}}\left(\bmod p^{c^{\prime}+1}\right)$, or $e \cdot u \equiv 0$ $(\bmod p)$. This is a contradiction, since $(e, p)=1$ and $(u, p)=1$.

(2) If $|Z(G)|=1$, then every power of $b$ induces a nontrivial automorphism of $\langle a\rangle$, so we have a monomorphism of $\langle b\rangle$ into $\operatorname{Aut}\left(S_{p}(G)\right)$. Since $\left|\operatorname{Aut}\left(S_{p}(G)\right)\right|=p^{c-1}(p-1)$, and $(e, p)=1$, we conclude that $e$ divides $p-1$.

(3) Assume $|Z(G)|=1$, and let $H$ be a subgroup of $G$. If $H$ is of $p$-order it is a subgroup of a cyclic group. If it is of $p^{\prime}$-order there is an automorphism of the group carrying it into $\langle b\rangle$, so it is also a subgroup of a cyclic group. If $H$ is of mixed order, then we may assume that it has a $p^{\prime}$ element $b^{\prime}$ of maximal order and a $p$-element $a^{m^{\prime \prime}}$ of maximal order. Then $H$ is a semidirect product of $\left\langle a^{m^{\prime \prime}}\right\rangle$ by $\left\langle b^{\prime}\right\rangle$. The center is trivial because there is an automorphism of $G$ carrying $b^{\prime}$ into a power of $b$, and every element of $\langle b\rangle$ induces a nontrivial automorphism of $\langle a\rangle$, as in the proof of (2).

(4) It was shown in Curtis and Reiner [1, page 301], for $k=\mathbb{C}$, that $H^{2}\left(G, k^{*}\right) \stackrel{\sim}{\rightarrow} C_{q}$, with $q=\operatorname{gcd}(m, r-1) \operatorname{gcd}\left(m,\left(r^{e}-1\right) /(r-1)\right) / m$. Since we showed in the proof of (1) that $r-1$ is not divisible by $p$, we conclude 
that $\operatorname{gcd}(m, r-1)=1$ and $\operatorname{gcd}\left(m,\left(r^{e}-1\right) /(r-1)\right)=m$, so $q=1$, and $H^{2}\left(G, k^{*}\right)$ is trivial. The proof in [1] actually holds true for any algebraically closed field of characteristic not equal to $p$.

Definition. A field $k$ of characteristic $p$ will be called $p$-sufficiently large if it contains all $|G|_{p^{\prime}}$-roots of unity and all $s$ roots of unity for $s<$ $|G|_{p},(s, p)=1$.

REMARK. If $\pi$ is any prime in $O_{S}$ lying over $p$, then $O_{S} /(\pi)$ will be $p$-sufficiently large.

Let $F$ be an algebraically closed field of characteristic prime to $|G|$.

Definition. For a finite group $G$, a $p$-modular semisimple deformation over a field $k$ is a deformation of $k G$ to a semisimple algebra $\bigoplus M_{d}(k)$, where the degrees $d$ of the various matrix blocks are the same as those of $F G$.

In [10], we proved that any group with cyclic $p$-Sylow group has a $p$ modular semisimple deformation for any $p$-sufficiently large field. However, in the case of $p-p^{\prime}$ metacyclic groups with trivial center, the deformation can be constructed explicitly without reference to sophisticated block theory, and we now make this construction.

We first describe $F G$ for char $F$ relatively prime to $p$. This is a standard textbook exercise in character theory. The commutator subgroup $G^{\prime}$ of $G$ is $P$ because $Z(G)=1$. Since $\left|G / G^{\prime}\right|=e, G$ has $e$ linear characters. In addition, if we let $n=(m-1) / e$, there are $n$ conjugacy classes of nontrivial characters of $P$, each of which induces an irreducible character of degree $e$. Summing degrees we have

$$
e+n \cdot e^{2}=e(1+n e)=e \cdot m=|G|,
$$

so these are all irreducible characters. The group algebra is thus

$$
F G \stackrel{\sim}{\rightarrow} F^{e} \times \prod^{n} M_{e}(k) .
$$

The structure of $k G$ for $\operatorname{char} k=p$ was determined by Morita in 1951 in [8], but we give a different presentation which is a preparation for the remainder of the proof.

By a theorem of Wallace [11], we have (Karpilovsky [6, page 193]) that $\operatorname{dim} J(k G)=\operatorname{dim} G-e=e^{2} n$. Also $J(k G)$ is principally generated by $(1-a)$ for any generator $a$ of the $p$-Sylow subgroup (Karpilovsky [6, page 299]). We can define $e$ orthogonal idempotents

$$
\varepsilon_{i}=(1 / e) \sum_{j=0}^{e-1} r^{i j} b^{j}, \quad i=0, \ldots, e-1 .
$$


Here $r$ is the integer appearing in the presentation of the metacyclic group. Note that $r$ is an $e$ th root of unity, modulo $p$, since $r^{e} \equiv 1(\bmod m)$. We showed in (3) of Lemma 1 that $r$ is a primitive root of unity modulo $m=p^{c}$. Thus, $r$ is a primitive $e$ th root of unity modulo $p$, for if $r^{e^{\prime}} \equiv 1$ $(\bmod p)$ for $e^{\prime}$ properly dividing $e$, then we could write $r^{e^{\prime}}=u p^{c^{\prime}}+1$ for $u$ with $\operatorname{gcd}(u, p)=1$ and $c^{\prime}<c$. Let $e^{\prime \prime}=e / e^{\prime}$. Then $1 \equiv r^{e^{\prime} e^{\prime \prime}}=\left(u p^{c^{\prime}}+1\right)^{e^{\prime \prime}}$ $\left(\bmod p^{c}\right)$. Since $c^{\prime}<c, 1 \equiv u e^{\prime \prime} p^{c^{\prime}}+1\left(\bmod p^{c^{\prime}+1}\right)$, so $u e^{\prime \prime} \equiv 0(\bmod p)$. Since $(u, p)=1$ and $\left(e^{\prime \prime}, p\right)=1$, this is a contradiction.

The idempotents $\varepsilon_{0}, \ldots, \varepsilon_{e-1}$ form a basis for $k G / J(k G)$ and a basis for $J(k G)$ is given by powers of any generator times elements of a basis of $k G / J(k G)$. Therefore if $x$ is any generator of $J(k G)$, the elements $\varepsilon_{i} \cdot x^{j}$ form a basis for $J(k G)$.

Claim. Put $z=(1 / e)\left(\sum_{i=0}^{e-1} r^{-i}\left(1-a^{r^{i}}\right)\right)$. Then $z$ is a generator of $J(k G)$.

Proof of Claim. We first note that in fact $z=(1 / e) \sum_{i=0}^{e-1}\left(-r^{-i}\right) a^{r^{i}}$, since the sum of all distinct powers of a root of unity is zero. Let $P=S_{p}(G)$. It suffices to prove that $z$ is a generator of $J(k P)$ [6, page 299]. For any integer $q,\left(1-a^{q}\right) \equiv q(1-a) \bmod J(k P)^{2}$, since

$$
a^{q}=(1-(1-a))^{q} \equiv 1-q(1-a) \bmod (J P)^{2} .
$$

Thus

$$
q(1-a) \equiv 1-a^{q} \bmod J(k P)^{2}
$$

If $q \not \equiv 0 \bmod p$, then

$$
(1-a) \equiv q^{-1}\left(1-a^{q}\right) \bmod (J P)^{2}
$$

Taking an average over all $q$ of the form $q=r^{j}$, we have

$$
\left.z=(1 / e) \sum\left(r^{-j}\right)\left(1-a^{r^{j}}\right)\right) \equiv(1-a) \bmod J(k P)^{2}
$$

Since $(1-a)$ is a generator for $J(k P)$, so is $z$. This proves the claim. 
We now use $z$ to construct an easily deformable multiplication table for $k G$. We have

$$
\begin{aligned}
\varepsilon_{0}(1-a) \varepsilon_{1}= & \varepsilon_{0} \cdot \varepsilon_{1}-\varepsilon_{0} a \varepsilon_{1}=-\varepsilon_{0} a \varepsilon_{1} \\
= & (1 / e)\left(1+b+\cdots+b^{e-1}\right)(-a)(1 / e)\left(1+r b+\cdots+r^{e-1} b^{e-1}\right) \\
= & -\left(1 / e^{2}\right)\left(a+a^{r} b+\cdots+a^{r^{e-1}} b^{e-1}\right)\left(1+r b+\cdots+r^{e-1} b^{e-1}\right) \\
= & -\left(1 / e^{2}\right)\left(a+r^{-1} a^{r}+r^{-2} a^{r^{2}}+\cdots+r^{-e+1} a^{r^{e-1}}\right) \\
& \cdot\left(1+r b+\cdots+r^{e-1} b^{e-1}\right) \\
= & -(1 / e)\left(\sum_{i=0}^{e-1} r^{-i} a^{r^{r}}\right) \varepsilon_{1} \\
= & z \varepsilon_{1}-(1 / e)\left(\sum_{i=0}^{e-1} r^{-i}\right) \varepsilon_{1} \\
= & z \varepsilon_{1} .
\end{aligned}
$$

A slight generalization of the calculation done above will show that for any $i$,

$$
\varepsilon_{i}(1-a) \varepsilon_{i+1}=z \varepsilon_{i+1} \text {. }
$$

Dually, we have

$$
\begin{aligned}
\varepsilon_{i}(1- & a) \varepsilon_{i+1}=\varepsilon_{i} \cdot \varepsilon_{i+1}-\varepsilon_{i} a \varepsilon_{i+1}=-\varepsilon_{i} a \varepsilon_{i+1} \\
= & (1 / e)\left(1+r^{i} b+\cdots+r^{i(e-1)} b^{e-1}\right)(-a) \\
& \cdot(1 / e)\left(1+r^{i+1} b+\cdots+r^{(i+1)(e-1)} b^{e-1}\right) \\
= & -\left(1 / e^{2}\right)\left(1+r^{i} b+\cdots+r^{i(e-1)} b^{e-1}\right) \\
& \cdot\left(a+r^{i+1} b a^{r^{-1}}+\cdots+r^{(e-1)(i+1)} b^{e-1} a^{r^{-(e-1)}}\right) \\
= & -(1 / e) \varepsilon_{i}\left(\sum r^{i} a^{r^{-i}}\right) \\
= & \varepsilon_{i} z .
\end{aligned}
$$

Furthermore, since $1=\varepsilon_{0}+\cdots+\varepsilon_{e-1}$, we have

$$
z=\varepsilon_{0}(1-a) \varepsilon_{1}+\varepsilon_{1}(1-a) \varepsilon_{2}+\cdots+\varepsilon_{e-1}(1-a) \varepsilon_{0},
$$

and more generally,

$$
z^{j}=\varepsilon_{0} z^{j} \varepsilon_{j}+\cdots+\varepsilon_{e-1} z^{j} \varepsilon_{e-1+j}
$$

Since $J(k P)^{m}=0$, we know that $z^{m}=0$. Thus we have a basis $\left\{\varepsilon_{i} z^{j}\right\}$ for $J(k G)$ such that each basis element lies in a single component of the Pierce decomposition $\bigoplus \varepsilon_{i} k G \varepsilon_{j}$ of $k G$ with respect to the orthogonal idempotent 
set $\varepsilon_{0}, \ldots, \varepsilon_{e-1}$. The multiplication table of the algebra is given by

$$
\left(\varepsilon_{i} z^{j}\right)\left(\varepsilon_{i^{\prime}} z^{l}\right)= \begin{cases}0, & \text { if } i+j \not \equiv i^{\prime} \bmod e \text { or } j+l \geq m, \\ \varepsilon_{i} z^{j+l}, & \text { if } i+j \equiv i^{\prime} \bmod e \text { and } j+l<m .\end{cases}
$$

REMARK. The quiver $Q$ of $k G$ is the directed graph with $e$ points $\varepsilon_{0}$, $\ldots, \varepsilon_{e-1}$ and $e$ arrows $\omega_{0}, \ldots, \omega_{e-1}$ with each $\omega_{i}$ going from $\varepsilon_{i}$ to $\varepsilon_{i+1}$, and $k G$ is the quotient of the path algebra $k[Q]$ of the quiver $Q$ by the ideal generated by

$$
\left(\omega_{i} \omega_{i+1} \cdots \omega_{e-1} \omega_{0} \cdots \omega_{i-1}\right)^{n} \omega_{i}=0, \quad \text { for } i=0, \ldots, e-1 .
$$

Definition. For $q \in \mathbb{Z} / m \mathbb{Z}$, define an equivalence relation $\sim$ by $q \sim l$ if and only if there is a number $s$ such that $l \equiv q r^{s}(\bmod m)$. The equivalence class of $q$ is

$$
[q]=\left\{q, q r, \ldots, q r^{e-1}\right\},
$$

since $r^{e} \equiv 1(\bmod m)$. Also $a^{q}$ is a conjugate to $a^{l}$ if and only if $q \sim l$.

Having described the structure of $k G$, we now exhibit the deformation of $k G$ to $k^{e} \times \prod^{n} M_{e}(k)$. We begin with a standard basis $E_{0}, \ldots, E_{e-1},\left\{E_{i j}^{[q]}\right\}$, $i, j=0, \ldots, e$, for the semisimple algebra. The primitive idempotents are $E_{0}, \ldots, E_{e-1}$, and $\left\{E_{i i}^{[q]}\right\}, i=1, \ldots, e$. Let $\xi$ be a primitive $n$th root of unity. Choose an arbitrary representative $q$ of each equivalence class $[q]$. For each representative $q$, choose $\xi_{q}$ to be a power of $\xi$, in such a way that $\left\{\xi_{q}^{e}\right\}_{[q]}$ are distinct. Define the elements

$$
\begin{gathered}
\tilde{\varepsilon}_{i}=E_{i}+\sum_{[q]} E_{i i}^{[q]}, \\
\tilde{\omega}_{i}=T\left(\sum_{[q]} \xi_{q} E_{i i+1}^{[q]}\right), \\
\tilde{z}=\tilde{\omega}_{0}+\cdots+\tilde{\omega}_{e-1},
\end{gathered}
$$

where $T$ is an indeterminate. The $\tilde{\varepsilon}_{i}$, being sums of orthogonal idempotents, are also idempotents. When $T \neq 0$, we get $|k G|$ elements $\left\{\tilde{\varepsilon}_{i} \tilde{z}^{j}\right\}$, for $j=0, \ldots$, en. We claim that these elements are in fact linearly independent, and therefore form a basis of $k G$ since in any given Pierce component $\tilde{e}_{i} \tilde{A}_{\tilde{e}}$ we get $n$ radical elements of the form

$$
\tilde{e}_{i} \tilde{z}^{j-i+l e}=T^{j-i+l e} \sum_{[q]} \xi_{q}^{(j-i+l e)} E_{i j}^{[q]}, \quad \text { for } l=0, \ldots, n-1 .
$$

Consider the matrix of coefficients $\left[T^{(j-i+l e)} \xi^{j-i+l e}\right]$. After dividing each column $q$ by $T^{j-i} \xi_{q}^{j-i}$, and each row $l$ by $T^{l e}$, we are left with a Vandermonde matrix $\left[\left(\xi_{q}^{e}\right)^{l}\right]$, whose determinant is nonzero because the elements $\xi_{q}^{e}$ 
are distinct. Thus for $T \neq 0$ the given elements form a basis. Furthermore

$$
\tilde{\varepsilon}_{i} \tilde{z}^{e n}=T^{e n} \sum_{[q]} \xi_{q}^{e n} E_{i i}^{[q]}=T^{e n} \sum_{[q]} E_{i i}^{[q]},
$$

since $\xi_{q}^{e n}=1$. Therefore $\left(\tilde{\varepsilon}_{i} \tilde{z}^{e n}\right)\left(\tilde{\varepsilon}_{i} \tilde{z}\right)=T^{e n} \tilde{\varepsilon}_{i} \tilde{z}$.

The multiplication table is given by

$$
\left(\tilde{\varepsilon}_{i} \tilde{z}^{j}\right)\left(\tilde{\varepsilon}_{i^{\prime}} \tilde{z}^{l}\right)= \begin{cases}0, & \text { if } i+j \not \equiv i^{\prime}, \\ T^{e n} \tilde{\varepsilon}_{i} \tilde{z}^{j+l-e n}, & \text { if } i+j \equiv i^{\prime} \text { and } j+l \geq m, \\ \tilde{\varepsilon}_{i} \tilde{z}^{j+l}, & \text { if } i+j \equiv i^{\prime} \text { and } j+l<m .\end{cases}
$$

When $T=0$ this reduces to the multiplication table of $k G$.

Before continuing to describe the analogy between the split integral group ring and the $p$-modular semisimple deformation, we present in considerable detail the elementary example which served as motivation for the calculations in the theory.

EXAMPLe 2. Consider the symmetric group

$$
S_{3}=\left\langle a, b \mid a^{3}=1, b^{2}=1, b a b=a^{2}\right\rangle .
$$

This is the simplest example of a metacyclic 3-3 group. Over a sufficiently large field of characteristic other then 2 or 3 , there are two isolated idempotents and one $2 \times 2$ matrix block.

The idempotents $\varepsilon_{0}$ and $\varepsilon_{1}$ defined above are given by

$$
\varepsilon_{0}=(1+b) / 2, \quad \varepsilon_{1}=(1-b) / 2 .
$$

The element $z$ is given by $z=\left(a-a^{2}\right) / 2$. The basis for the 3 -modular algebra is given by $B=\left\{\varepsilon_{0}, \varepsilon_{1}, \varepsilon_{0} z, \varepsilon_{1} z_{1}, \varepsilon_{0} z^{2}, \varepsilon_{1} z_{2}\right\}$ where

$$
\varepsilon_{0} z^{2}=(1+b)\left(a+a^{2}-2\right) / 8, \quad \varepsilon_{1} z^{2}=(1-b)\left(a+a^{2}-2\right) / 8 .
$$

The same formulae, with coefficients from $O_{S}$ instead of from a modular field $k$, determine well-defined elements of $O_{S}\left[S_{3}\right]$. Since the elements of the basis $B$ are linearly independent at the special prime $\pi$ lying over $p$, they must be linearly independent at almost all primes of $O_{S}$.

At primes which do not divide 3 , we have two orthogonal idempotents $f_{0}=\left(1+a+a^{2}\right) / 3$ and $f_{1}=\left(2-a-a^{2}\right) / 3$, where $f_{1}$ is the central idempotent of the $2 \times 2$ matrix block. Direct computation gives

$$
z^{2}=(-3 / 4) f_{1} \text {. }
$$

Taking the square root of the constant, we have $\sqrt{-3 / 4}= \pm i \sqrt{3} / 2=$ $\pm\left(\omega-\omega^{2}\right) / 2$. Set $T_{1}=\left(\omega-\omega^{2}\right) / 2$. Then $z^{2}=T_{1}^{2} \cdot f_{1}$. Furthermore, since $f_{0} z=0$ and $f_{1} z=z$, we have

$$
z^{3}=T_{1}^{2} z
$$


which is exactly the equation that appears in the 3-modular semisimple deformation. Since

$$
z=\left(a-a^{2}\right) / 2 \text { and } T_{1}=\left(\omega-\omega^{2}\right) / 2,
$$

the number $T_{1}$ (which we will later call a "pseudoparameter") is obtained from $z$ by substituting the cubed root of unity $\omega$ for the cubed root $a$ of the identity in the group.

The quiver $Q$ of $k[G]$ is given by two points, $\varepsilon_{0}$ and $\varepsilon_{1}$, and two arrows $x_{01}$ from $\varepsilon_{0}$ to $\varepsilon_{1}$ and $x_{10}$ from $\varepsilon_{1}$ to $\varepsilon_{0}$. The path algebra $k(Q)$ of $Q$ is generated by $x_{01}$ and $x_{10}$. The modular group algebra is the quotient of this path algebra by the ideal generated by $x_{10} x_{01} x_{10}$ and $x_{01} x_{10} x_{01}$.

The $k$-modular semisimple deformation is the quotient of $K[T](Q)$ by the ideal generated by relations

$$
x_{01} x_{10} x_{01}-T^{2} x_{01}=0, \quad x_{10} x_{01} x_{10}-T^{2} x_{10}=0 .
$$

The split integral group ring is isomorphic to the quotient of the path algebra $O_{S}(Q)$ by the relations

$$
x_{01} x_{10} x_{01}-T_{1}^{2} x_{01}=0, \quad x_{10} x_{01} x_{10}-T_{1}^{2} x_{10}=0 .
$$

We can obtain matrix units for the $2 \times 2$ block by setting

$$
\begin{aligned}
& E_{00}=\varepsilon_{0} f_{1}, \quad E_{01}=\varepsilon_{0}\left(z / T_{1}\right), \\
& E_{10}=\varepsilon_{1}\left(z / T_{1}\right), \quad E_{11}=\varepsilon_{1} f_{1} .
\end{aligned}
$$

Over the prime $\pi$, the cubed roots of unity all become equal: $\omega=\omega^{2}=1$. Thus over the prime $\pi, T_{1}$ reduces to zero, so $E_{10}$ and $E_{01}$ are not well defined. Also $E_{00}$ and $E_{11}$ are not well-defined over $\pi$ because $f_{1}$ has denominator 3 .

\section{The metacyclic function}

Combining the results on the structure of metacyclic p-groups with Example 2 above, we are led to the following function.

DEFINITION. Let $G$ be a metacyclic group with presentation

$$
\left\langle a, b \mid a^{m}=1, b^{e}=1, b a b^{-1}=a^{r}\right\rangle,
$$

for $m=p^{c}$. Let $\pi$ be a prime lying over $p$ in $O_{S}$. Let $\eta$ be the primitive $e$ th root of unity in $O_{S}$ which is congruent to $r$ modulo the distinguished prime $\pi$. The metacyclic function is

$$
\psi(y, x)=(1 / e) \sum_{s=0}^{e-1} y^{-s} x^{r^{s}},
$$


where $y$ is a power of $\eta$, and $x$ is a power either of $a$ or of $\omega$, for a primitive $m$ th root of unity $\omega$ in $O_{S}$.

We now consider the relationship between the metacyclic function and the block idempotents of $G$ for nonmodular primes. Recall the equivalence relation on $\mathbb{Z} / m \mathbb{Z}$ defined earlier by multiplication by powers of $r$.

Let $P=\langle a\rangle$ be the $p$-Sylow subgroup of $G$. Two characters $a \mapsto \omega^{q}$ and $a \mapsto w^{l}$ are conjugate if and only if $q \sim l$, so the induced characters in $G$ are indexed by the equivalence classes $[q]$. Let $f^{[q]}$ be the central idempotent corresponding to the class $q$. Now $f^{[q]}$ is the sum of the idempotents in $P$ corresponding to the various linear characters, so

$$
\begin{aligned}
f^{[q]} & =(1 / m) \sum_{j=0}^{m-1} \sum_{s=0}^{e-1} \omega^{-q r^{s} j} a^{j} \\
& =(e / m) \sum_{j=0}^{m-1} \psi\left(1, \omega^{-q j}\right) a^{j}
\end{aligned}
$$

Define $f^{0}=(1 / m) \sum_{j=0}^{m-1} a^{j}$ to be the principal block idempotent of $P$, so that

$$
f^{0}=1-\sum_{[q]} f^{[q]}
$$

We now calculate those properties of the metacyclic function which will be needed to put the split integral deformation into the same form as the $p$-modular semisimple deformation. We first define $\varepsilon_{s}$ in $O_{S} G$ by

$$
\varepsilon_{s}=1+\eta^{s} b+\cdots+\eta^{s(e-1)} b^{e-1}, \quad s=0, \ldots, e-1 .
$$

Proposition 1. The metacyclic function $\psi(y, x)$ satisfies the following properties:

(1) $\varepsilon_{s} \psi\left(y, a^{j}\right)=\psi\left(y, a^{j}\right) \varepsilon_{s+1}=\varepsilon_{s} \psi\left(y, a^{j}\right) \varepsilon_{s+1}$;

(2) $\psi\left(y, x^{r^{3}}\right)=y^{s} \psi(y, x)$;

(3) $f_{0} \psi(\eta, a)=0$;

(4) $f^{[q]} \psi(\eta, a)=\psi\left(\eta, \omega^{q}\right)(e / m) \sum_{j=0}^{e-1} \psi\left(\eta^{-1}, \omega^{-q j}\right) a^{j}$.

Proof. (1) This is just the calculation made above for $\varepsilon_{s}$ and $z$, with $\eta$ substituted for $r$ where appropriate. 
(2)

$$
\begin{aligned}
\psi\left(y, x^{r^{s}}\right) & =(1 / e) \sum_{l=0}^{e-1} y^{-l} x^{r^{s} r^{l}} \\
& =(1 / e) y^{s} \sum_{l=0}^{m-1} y^{-(s+l)} x^{r^{s+l}} \\
& =y^{s} \psi(y, x) .
\end{aligned}
$$

(3)

$$
\begin{aligned}
f^{0} \cdot \psi(\eta, a) & =\left[(1 / m) \sum_{i=0}^{m-1} a^{i}\right]\left[(1 / e) \sum_{s=0}^{e-1} \eta^{-s} a^{r^{s}}\right] \\
& =(e / m) \sum_{j=0}^{m-1}\left(\sum_{s=0}^{e-1} \eta^{-s}\right) a^{j}=0 .
\end{aligned}
$$

(4)

$$
\begin{aligned}
f^{[q]} \psi(\eta, a) & =\left[(e / m) \sum_{i=0}^{m-1} \psi\left(i, \omega^{q i}\right) a^{i}\right]\left[(1 / e) \sum_{s=0}^{e-1} \eta^{-s} a^{r^{s}}\right] \\
& =(1 / m) \sum_{i=0}^{m-1}\left(\sum_{s=0}^{e-1}(1 / e) \sum_{l=0}^{e-1} \eta^{-s} \omega^{-q r^{l} i}\right) a^{i+r^{s}} \\
& =(1 / m) \sum_{j=0}^{m-1}\left(\sum_{l=0}^{e-1}(1 / e) \sum_{s=0}^{e-1} \eta^{-s} \omega^{q r^{s+l}} \omega^{-q r^{l} j}\right) a^{j} \\
& \left.\left.=(1 / m) \sum_{j=0}^{m-1}\left(\sum_{l=0}^{e-1} \psi\left(\eta, \omega^{q r^{l}}\right) \omega^{-q r^{l} j}\right) a^{j}\right) a^{s}\right) \\
& =(1 / m) \sum_{j=0}^{m-1}\left(\sum_{l=0}^{e-1} \eta^{l} \psi\left(\eta, \omega^{q}\right) \omega^{-q r^{l} j}\right) a^{j} \\
& =\psi\left(\eta, \omega^{q}\right)(1 / m) \sum_{j=0}^{m-1}\left(\sum_{l=0}^{e-1}\left(\eta^{l} \omega^{-q r^{l} j}\right)\right) a^{j} \\
& =\psi\left(\eta, \omega^{q}\right)(e / m) \sum_{j=0}^{m-1} \psi\left(\eta^{-1}, \omega^{-q j}\right) a^{j} .
\end{aligned}
$$

In order to make our notation compatible with that of the $p$-modular deformation, we now introduce the following notation: 
Definition. Set

$$
\begin{gathered}
\hat{Z}=\psi(\eta, a), \quad \hat{T}=\psi(\eta, \omega), \quad \hat{\xi}_{q}=\psi\left(\eta, \omega^{q}\right) / \psi(\eta, \omega), \\
y_{q}=(e / m) \sum_{j=0}^{m-1} \psi\left(\eta^{-1}, \omega^{-q j}\right) a^{j} .
\end{gathered}
$$

We now come to our main result:

Proposition 2. (a) The elements

$$
E_{i j}^{q}=\varepsilon_{i} y_{q}^{s} \varepsilon_{j}, \quad i \neq j, \quad i+s \equiv j(\bmod e)
$$

and

$$
E_{i i}^{q}=\varepsilon_{i} f^{[q]}
$$

form a set of matrix units for the block with idempotent $f^{[q]}$ over nonmodular primes.

(b) The elements $\varepsilon_{i} \widehat{Z}^{s}$ for $i=0, \ldots, e-1, s=0, \ldots$, en form a basis for the group algebra, for almost all primes.

(c) For nonmodular primes the basis element can be written in the form

$$
\varepsilon_{i} Z^{s}=\sum_{[q]} \hat{\xi}_{q}^{s} \hat{T}^{s} E_{i i+s}^{q}, \quad s=0, \ldots, e n, \quad i=0, \ldots, e-1 .
$$

Proof. Since $\varepsilon_{0}, \ldots, \varepsilon_{e-1}$ is a set of orthogonal idempotents, in order to verify the relations among the matrix units it suffices to prove that $y_{q}^{e}=f^{[q]}$.

We prove by injunction that for $h \geq 1$,

$$
y_{q}^{h}=(e / m) \sum_{j=0}^{m-1} \psi\left(\eta^{h}, \omega^{-q j}\right) a^{j} .
$$

For $h=1$ this is the definition of $y_{q}$. Suppose it has been proven for $h-1$. Then

$$
\begin{aligned}
y_{q}^{h} & =y_{q} \cdot y_{q}^{h-1}=\left[(e / m) \sum_{i=0}^{m-1} \psi\left(\eta, \omega^{-q i}\right) a^{i}\right]\left[(e / m) \sum_{j=0}^{m-1} \psi\left(\eta^{h-1}, \omega^{-q j}\right) a^{j}\right] \\
& =\left(1 / m^{2}\right) \sum_{i=0}^{m-1} \sum_{l=0}^{e-1} \sum_{j=0}^{m-1} \sum_{s=0}^{e-1} \eta^{-l} \omega^{-q i r^{l}} \eta^{-(h-1) s} \omega^{-q j r^{s}} a^{i+j} \\
& =\left(1 / m^{2}\right) \sum_{l=0}^{e-1} \sum_{j^{\prime}=0}^{m-1} \sum_{s=0}^{e-1} \eta^{-l} \eta^{-(h-1) s} \omega^{-q j^{\prime} r^{l}}\left(\sum_{j=0}^{m-1} \omega^{-q j\left(r^{s}-r^{l}\right)}\right) a^{j^{\prime}} .
\end{aligned}
$$


Now $\sum^{m-1} \omega^{-q j\left(r^{s}-r^{l}\right)}$ equals 0 if $r^{s} \neq r^{l}$ and equals $m$ if $r^{s}=r^{l}$. Thus all terms except those with $l=s$ drop out, and we cancel $m / m^{2}=1 / m$. Thus

$$
\begin{aligned}
y_{q}^{h} & =(1 / m) \sum_{j^{\prime}=0}^{m-1} \sum_{s=0}^{e-1} \eta^{-s} \eta^{-(h-1) s} \omega^{-q j^{\prime} r^{\prime}} a^{j^{\prime}} \\
& =(1 / m) \sum_{j=0}^{m-1} \sum_{s=0}^{e-1} \eta^{-h s} \omega^{-q j^{\prime} r^{s}} a^{j^{\prime}} \\
& =(e / m) \sum_{j^{\prime}=0}^{m-1} \psi\left(\eta^{h}, \omega^{-q j^{\prime}}\right) a^{j^{\prime}}
\end{aligned}
$$

as required.

The elements $\varepsilon_{i} \widehat{Z}^{s}$ are linearly independent at the prime $\pi=0$, and therefore almost everywhere. Now $f^{0} \hat{Z}=0$ implies that $\left(\sum f^{[q]}\right) \widehat{Z}=\widehat{Z}$, and thus

$$
\left(\sum f^{[q]}\right) \varepsilon_{i} \hat{Z}^{s}=\varepsilon_{i} \widehat{Z}^{s}
$$

However

$$
\begin{aligned}
f^{[q]} \varepsilon_{i} \hat{Z}^{s} & =\varepsilon_{i} f^{[q]} \hat{Z}^{s}=\varepsilon_{i}\left(f^{[q]} \hat{Z}\right)^{s}=\varepsilon_{i}\left(\psi\left(\eta, \omega^{q}\right) y_{q}\right)^{s} \\
& =\varepsilon_{i} \psi\left(\eta, \omega^{q}\right)^{s} y_{q}^{s}=\left(\psi\left(\eta, \omega^{q}\right)\right) n^{s}\left(\varepsilon_{i} y_{q}^{s}\right) \\
& =\left(\left(\psi\left(\eta, \omega^{q}\right) / \psi(\eta, \omega)\right)^{s} \cdot \psi(\eta, \omega)^{s}\right) \varepsilon_{i} y_{q}^{s} \\
& =\hat{\xi}_{q}^{s} \cdot \widehat{T}^{s} \cdot E_{i i+s}^{q} .
\end{aligned}
$$

Thus

$$
\begin{aligned}
\varepsilon_{i} \widehat{Z}^{s} & =\left(\sum f^{[q]}\right) \varepsilon_{i} \widehat{Z}^{s}=\sum_{[q]} f^{[q]} \varepsilon_{i} \hat{Z}^{s} \\
& =\sum_{[q]} \hat{\xi}_{q}^{s} \cdot \widehat{T}^{s} E_{i i+s}^{q} .
\end{aligned}
$$

Note that the choice of a representative $q$ of the class $[q]$ causes differences in the values of $\hat{\xi}_{q}^{s}$ and $E_{i i+s}^{q}$ which cancel out in the product.

Definition. A nonzero element $T$ of $O_{S}$ which is zero modulo the distinguished prime $\pi$ will be called a pseudo-parameter.

The elements $T_{q}=\psi\left(\eta, \omega^{q}\right) \equiv(1 / e) \sum_{l=0}^{e-1} \eta^{-l}=0(\bmod \pi)$, so all these elements $T_{q}$ are pseudo-parameters. The theory is particularly simple in the case $m=p$, and $e=p-1$. In that case there is only one matrix block, and $r$ is a generator for the multiplicative group of $\mathbb{Z} / m \mathbb{Z}$, so that there is a single equivalence class [1]. If we choose a representative $q=r^{s}$, then

$$
\psi\left(\eta, \omega^{q}\right)=\psi\left(\eta, \omega^{r^{s}}\right)=\eta^{s} \psi(\eta, \omega)
$$


Thus in this case,

$$
\xi_{q}=\psi\left(\eta, \omega^{q}\right) / \psi(\eta, \omega)=\eta^{s}
$$

In this case the $p$-modular semisimple deformation and the split integral group ring are entirely analogous.

At the other extreme, consider the case of a cyclic $p$-group, with $e=1$, in which each equivalence class $[q]$ contains only $q$. The elements $\psi\left(\eta, \omega^{q}\right)$ for $\eta=1, e=1$ are just $m$ th roots of unity $\omega^{q}$, so

$$
\xi_{q}=\psi\left(\eta, \omega^{q}\right) / \psi(\eta, \omega)=\omega^{q-1}, \quad q=0, \ldots, m-1 .
$$

This shows that we cannot in general expect the $\xi_{q}$ to be $(m-1)$ th roots of unity.

We want to be able to describe the relations on the path algebra $O_{S}(Q)$ in the general case when the $\xi_{q}$ are not $(m-1)$ th roots of unity. We must therefore introduce the following notation:

Definition. Let $R$ be a commutative ring. Let $Q$ be a quiver which is a cycle of length $e$ with vertices $\varepsilon_{0}, \ldots, \varepsilon_{e-1}$ and arrows $x_{01}, \ldots, x_{(e-1) 0}$. Standard circuit relations of weight $n$ on the path algebra $R[Q]$ are given by setting

$$
x=x_{01}+\cdots+x_{(e-1) 0},
$$

fixing elements $\zeta \in R^{n}, T \in R$, and giving $e$ relations

$$
\varepsilon_{i} x \prod_{j=1}^{n}\left(x^{e}-\zeta_{j} T^{e}\right)=0 \text {. }
$$

Proposition 3. Let $G$ be a metacyclic p-p' group,

$$
G=\left\langle a, b \mid a^{m}=1, b^{e}=1, b a b^{-1}=a^{r}\right\rangle,
$$

with $m=p^{c}$ and $n=(m-1) / e$. Both the split integral group ring and the p-modular semisimple deformation are given by standard circuit relations on a quiver $Q$ which is a cycle of length $e$. For the split integral group ring the parameter ring is $O_{S}$ and the element $t$ is $\widehat{T}$. For the p-modular semisimple deformation the parameter ring is $k[T]$ and the element $t$ is just the indeterminate $T$.

Proof. For the $p$-modular semisimple deformation, the deformation is given by standard circuit relations of the form

$$
\varepsilon_{i} z \prod_{[q]}\left(z^{e}-\hat{\xi}_{q}^{e} T^{e}\right)=0,
$$

where the $q$ range over a set of representations of the equivalence classes $[q]$, and the $\xi_{q}$ is a primitive $e n$th root of unity. 
For the split integral group ring, we have

$$
\left(\varepsilon_{i} z^{e} \varepsilon_{i}\right)^{l}=\varepsilon_{i} z^{l e}=\sum \hat{\xi}_{q}^{e l} \cdot \widehat{T}^{e l} E_{i i}^{[q]}, \quad l=1, \ldots, n .
$$

The coefficients of the $E_{i i}^{[q]}$ form an $n \times n$ matrix

$$
\left[\hat{\xi}_{q}^{e l} \widehat{T}^{e l}\right] \text {. }
$$

Let us add a row of ones at the beginning. Each column $\left[1, \hat{\xi}_{q}^{e} T^{e}, \hat{\xi}_{q}^{2 e} T^{2 e}\right.$, $\left.\ldots, \hat{\xi}_{q}^{n e} T^{n e}\right]$ of the resulting matrix satisfies the equation

$$
h_{0} T^{n e}(1)+\cdots+h_{1} T^{e(n-1)}\left(\hat{\xi}_{q}^{e} T^{e}\right)+\cdots+h_{n}\left(\hat{\xi}_{q}^{n e} T^{n e}\right)=0,
$$

where $h_{0} T^{n e}, \ldots, h_{n} T^{0}$ are the coefficients of the polynomial $\prod_{[q]}\left(Y-\hat{\xi}_{q}^{e} T^{e}\right)$. Thus the coefficients give a linear dependence among the rows, implying that

$$
h_{0} T^{n e}\left(\sum E_{i i}^{q}\right)+h_{1} T^{(n-1) e}\left(\varepsilon_{i} z^{e}\right)+\cdots+h_{n} T^{0} \varepsilon_{i} z^{n e}=0 .
$$

Multiplying by $\varepsilon_{i} z$ and using the fact that $\varepsilon_{i} z\left(\sum E_{i i}^{q}\right)=\varepsilon_{i} z$, we get

$$
h_{0} T^{n e}\left(\varepsilon_{i} z\right)+h_{1} T^{(n-1) e} \varepsilon_{i} z^{e+1}+\cdots+h_{n} T^{0} \varepsilon_{i} z^{n e+1}=0 .
$$

In view of the source of the numbers $h_{i}$, we then have the standard circuit relation $\varepsilon_{i} z \prod_{[q]}\left(z^{e}-\xi_{q}^{e} T^{e}\right)=0$.

COROLLARY 3.1. Both the split integral group ring and the p-modular semisimple deformation are restrictions to subvarieties of $\operatorname{Spec}\left(O_{S}[T]\right)$ of a single unified deformation given by the following standard circuit relations on the path algebra $O_{S}[T][Q]$ :

$$
z \prod_{[q]}\left(z^{e}-\hat{\xi}_{q} \widehat{T}-\xi_{q} T\right)^{e} .
$$

Proof. Over the subscheme with ideal $\pi O_{S}[T], \hat{T}=0$; and over the subscheme with ideal $(T) O_{S}[T], T=0$.

COROLlaRY 3.2. Let $R=k[T]$. If $m=p$ and $e=p-1$, both the split integral group ring and the p-modular semisimple definition can be obtained from the respective path algebras $O_{S}[Q]$ and $R[Q]$ by relations

$$
\varepsilon_{i} z^{p}=\varepsilon_{i} t^{p-1} z,
$$

where in the integral case $t=(1 / e) \sum \eta^{-i} \omega^{r^{i}}=\psi(\eta, \omega)$, and in the $p$ modular case $t=T$.

EXAMPLE 3. Consider the group of order 20 which is the semidirect product of $C_{5}$ by $C_{4}$, with $m=5, e=4$ and $r=2$. Note that, as required, 
$2^{4} \equiv 1(\bmod 5)$. We calculate that $n=(m-1) / e=1$. Let $k$ have characteristic 5. The Cartan matrix, whose $i j$ entry is the number $\operatorname{dim} \varepsilon_{i}(k G) \varepsilon_{j}$, is then

$$
\left[\begin{array}{llll}
2 & 1 & 1 & 1 \\
1 & 2 & 1 & 1 \\
1 & 1 & 2 & 1 \\
1 & 1 & 1 & 2
\end{array}\right] .
$$

The modular group algebra $k G$ deforms to a semisimple algebra whose Car$\tan$ matrix is the direct sum of four $1 \times 1$ matrices and one $4 \times 4$ matrix. The four primitive idempotents in the group algebra decompose in pairs to give the eight idempotents of the semisimple algebra.

We now consider the split integral group ring. Since $O_{S}$ contains a fourth root of unity $i$, the prime $p=5$ splits into $\pi=(2-i)$ and $\pi^{\prime}=(2+i)$. We will work at the prime $\pi$, so that $\eta=i \equiv 2(\bmod \pi)$.

The four orthogonal idempotents are given by

$$
\begin{array}{ll}
\varepsilon_{0}=(1 / 4)\left(1+b+b^{2}+b^{3}\right), & \varepsilon_{1}=(1 / 4)\left(1+i b-b^{2}-i b^{3}\right), \\
\varepsilon_{2}=(1 / 4)\left(1-b+b^{2}-b^{3}\right), & \varepsilon_{3}=(1 / 4)\left(1-i b-b^{2}+i b^{3}\right) .
\end{array}
$$

The element $\widehat{Z}$ is given by

$$
\widehat{Z}=(1 / 4)\left(a-i a^{2}-a^{4}+i a^{3}\right) .
$$

Direct computation shows that

$$
\hat{Z}^{2}=((2 i-1) / 16)\left(a+a^{4}-a^{2}-a^{3}\right)=((-2-i) / 16 i)\left(a+a^{4}-a^{2}-a^{3}\right)
$$

and

$$
\widehat{Z}^{4}=-\left((2-i)^{2} / 256\right)\left(5-\left(1+a^{2}+a^{3}+a^{4}+a^{5}\right)\right)=-\left((2-i)^{2} / 256\right) \cdot 5 \cdot f^{[1]} \text {. }
$$

Let $\omega$ be a primitive fifth root of unity.

Since $\widehat{T}=(1 / 4)\left(\omega-i \omega^{2}-\omega^{4}+i \omega^{3}\right)$ is obtained from substituting $\omega$ for $a$, and $1+\omega+\omega^{2}+\omega^{3}+\omega^{4}=0$, we have

$$
\widehat{T}^{4}=-\left((2+i)^{2} / 256\right) 5 \text {. }
$$

Thus

$$
\hat{Z}^{4}=\widehat{T}^{4} \cdot f^{[1]} \text { and } \widehat{Z}^{5}=\widehat{T}^{4} \cdot \hat{Z} .
$$

The matrix units are generated by

$$
\begin{aligned}
E_{j j+1} & =\varepsilon_{i}(\hat{Z} / \hat{T}) \\
& =\frac{1}{4}\left(1+i^{j} b+i^{2 j} b^{2}+i^{3 j} b^{3}\right)\left(a-i a^{2}-a^{4}+i a^{3}\right) /\left(\omega-i \omega^{2}-\omega^{4}-i \omega^{3}\right) .
\end{aligned}
$$


EXAmple 4. If we let $G$ be the group of order 100 which is the semidirect product of $C_{25}$ by $C_{4}$, then the deformed algebra will be isomorphic to $k^{4} \times M_{4}(k)^{6}$. The Cartan matrix for $k G$ is

$$
\left[\begin{array}{llll}
7 & 6 & 6 & 6 \\
6 & 7 & 6 & 6 \\
6 & 6 & 7 & 6 \\
6 & 6 & 6 & 7
\end{array}\right]
$$

Since the Cartan matrix of the corresponding semisimple algebra is the direct sum of four $1 \times 1$ matrices and six $4 \times 4$ matrices, we see that again the Cartan matrix of the modular group algebra is obtained by stacking up the Cartan matrices of the nonmodular group algebra.

In this case we may take $r=7$. Once again $7 \equiv i(\bmod 2-i)$. The idempotents $\varepsilon_{0}, \ldots, \varepsilon_{s}$ are just as in Example 3 above. Taking $\omega$ to be a primitive 25 th root of unity, we now have

$$
\begin{gathered}
\hat{Z}=(1 / 4)\left(a+i a^{7}-a^{-1}+i a^{-7}\right), \\
\hat{T}=(1 / 4)\left(\omega-i \omega^{7}-\omega^{-1}+i \omega^{-7}\right), \\
\hat{\xi}_{q}=\left(\omega^{q}-i \omega^{7 q}-\omega^{-q}+i \omega^{-7 q}\right) /\left(\omega-i \omega^{7}-\omega^{-1}+i \omega^{-7}\right) .
\end{gathered}
$$

The six equivalence classes can be represented by $q=1,2,3,5,6$, and 9. The relations on the path algebra are then

$$
\widehat{Z} \prod_{[q]}\left(\hat{Z}^{4}-\hat{\xi}_{q}^{4} \widehat{T}^{4}\right) \text {. }
$$

At the prime $\pi$, where $\omega \equiv 1$ and $\widehat{T} \equiv 0$, the relations reduce to $\widehat{Z}^{25}=0$.

The matrix units of the matrix blocks are generated by

$$
E_{s s+1}^{q}=(1 / 25) \varepsilon_{s} \sum_{j=0}^{24} \psi\left(-i, \omega^{-q j}\right) a^{j}, \quad q=1,2,3,5,6,9 .
$$

The remaining 4 idempotents are $\varepsilon_{0} f^{0}, \varepsilon_{1} f^{0}, \varepsilon_{2} f^{0}, \varepsilon_{3} f^{0}$, where

$$
f^{0}=(1 / 25)\left(\sum_{j=0}^{24} a^{j}\right) \text {. }
$$

\section{General $p$-solvable groups of finite representation type}

We now turn to the general case of $p$-solvable groups of finite representation type. 
THEOREM. If $G$ is a p-solvable group of finite representation type, then for each p-block, the split integral group ring and the p-modular semisimple deformation are given by standard circuit relations.

Proof. We wish to reduce the general case to the $p-p^{\prime}$ metacyclic case dealt with above. We first note that if $G$ is $p$-solvable of finite representation type, then $G / O_{p^{\prime}}(G)$ is $p-p^{\prime}$ metacyclic with trivial center.

In order to decompose into blocks, we follow the proof of a theorem of Morita [6, page 240]. We let $N=O_{p^{\prime}}(G)$ and let $e_{1}, \ldots, e_{s}$ be a set of representatives of $G$-conjugacy classes of block idempotents of $N$. For any idempotent $e_{i}$, suppose $k N e_{i}$ is isomorphic to $M_{n_{i}}(K)$. The set of matrix units in $k N e_{i}$ is well defined at $\pi$ and therefore at almost every prime of $O_{S}$.

Let $e_{11}$ be one of the idempotents in this set of matrix units. Let $G_{i}$ be the inertia subgroup of $e_{i}$, that is, the centralizer of $e_{i}$, and let $n_{i}^{\prime}$ be the index $\left(G ; G_{i}\right)$. Let $e_{i}^{*}$ be the sum of the $n_{i}^{\prime} G$-conjugates of $e_{i}$. Then $e_{i}^{*}$ is a central idempotent in $G$.

At the prime $\pi$, we have

$$
k G e_{i}^{*} \stackrel{\sim}{\rightarrow} M_{n_{i}}\left(M_{n_{i}^{\prime}}\left(e_{11} k G_{i} e_{11}\right)\right) \stackrel{\sim}{\rightarrow} M_{n_{i}^{\prime} n_{i}}\left(e_{11} k G_{i} e_{11}\right) .
$$

Since the matrix units are well-defined at $\pi$, they are well-defined at almost every prime of $O_{S}$. Enlarging $O_{S}$ to $O_{S^{\prime}}$ if necessary to eliminate the bad primes, but with $O_{S^{\prime}} / \pi O_{S^{\prime}} \stackrel{\sim}{\rightarrow} O_{S} / \pi=k$, we will have

$$
O_{S^{\prime}} G_{i} e_{i}^{*} \stackrel{\sim}{\rightarrow} M_{n_{i}^{\prime} n_{i}}\left(e_{11} O_{S^{\prime}} G_{i} e_{11}\right) \text {. }
$$

In Schaps [9] it was shown that unicharacteristic deformation is independent of Morita equivalence class. The matrix units are rigid, and the deformation of the blocks depend on the deformations of $e_{11} k G e_{11}$. Since we have essentially the same matrix units for $O_{S^{\prime}} G_{i} e_{i}^{*}$, it suffices to show that the algebra $e_{11} k G_{i} e_{11}$ has a unicharacteristic deformation to a semisimple algebra which is analogous to $e_{11} O_{S^{\prime}} G_{i} e_{11}$.

In the proof of Morita's theorem (see Karpilovsky [6, page 240]) it is shown that for an algebraically closed field $\bar{k}$ of characteristic $0, e_{11} \bar{k} G_{i} e_{11}$ is isomorphic to $\bar{k}^{\alpha}\left(G_{i} / N\right)$, for some factor set $\alpha$. This part of the proof is actually independent of the hypothesis about characteristic or algebraic closure, so we in fact obtain such a factor set for $k$.

Carrying through the same analysis for the quotient field of $O_{S^{\prime}}$, and enlarging $O_{S^{\prime}}$ slightly if necessary, we may assume that we have a cocycle $\alpha^{\prime}$ such that

and that $\alpha^{\prime}$ reduces to $\alpha \bmod \pi$.

$$
e_{11} O_{S^{\prime}} G_{i} e_{11} \stackrel{\sim}{\rightarrow} O_{S^{\prime}}^{\alpha^{\prime}}\left(G_{i} / N\right)
$$


Now $G_{i} / N$ is a subgroup of $G / N$. Since $G / N$ is $p-p^{\prime}$ metacyclic with trivial center, so is $G_{i} / N$, by Lemma 1(4). The analysis on page 301 of Curtis and Reiner [1] shows that the cocycle $\alpha^{\prime}$ has finite order which is a power of $p$ in the cohomology group. Furthermore, if we attach a finite number of $p$-power roots to $O_{S^{\prime}}$, we may assume that $\alpha^{\prime}$ is given by roots of unity, and that $\alpha^{\prime}$, raised to its order, is actually 1 . The maximal possible order for $\alpha^{\prime}$ can then be calculated by the method on page 301 in Curtis and Reiner [1]. As shown in Lemma 1(4), for a $p$ - $p^{\prime}$ metacyclic group, $\alpha^{\prime}$ must actually be of order 1 , and thus identically equal to 1 . Thus we have in fact that

$$
e_{11} O_{S^{\prime}} G_{i} e_{11} \stackrel{\sim}{\rightarrow} O_{S^{\prime}}\left(G_{i} / N\right) \text { and } e_{11} k G_{i} e_{11} \stackrel{\sim}{\rightarrow} k\left(G_{i} / N\right)
$$

The analogy between the $p$-modular semisimple deformation and the integral group ring is then given by Propositions 2 and 3 in Section 4.

\section{References}

[1] C. Curtis and I. Reiner, Methods of representation theory I, Pure and Applied Mathematics, (John Wiley and Sons, 1981).

[2] F. Donald and F. Flanigan, 'A deformation theoretic version of Maschke's theorem for modular group algebras: The commutative case,' J. Algebra 29 (1974), 98-102.

[3] P. Gabriel, "Finite representation type is open", Representations of algebras, pp. 132155 (Lecture Notes in Math., 488, Springer Verlag, 1974).

[4] M. Gerstenhaber, 'On the deformations of rings and algebras,' Ann. of Math. (2) 79 (1964), 59-103.

[5] D. Happel, "Deformations of five dimensional algebras with units", Ring theory, (edited by F. van Oystaeyen, pp. 459-494) (Lecture Notes in Pure and Applied Mathematics, Marcel Dekker, 1979).

[6] G. Karpilovsky, The Jacobson radical group algebras, (North-Holland Mathematical Studies 135, 1987).

[7] S. Koshitani, 'On the nilpotency indices of the radicals of p-solvable groups,' Proc. Japan Acad. Ser. A 53 (1977), 13-16.

[8] $\mathrm{K}$. Morita, 'On group rings over a modular field which possess radicals expressible as principal ideals,' Sci. Rep. Tokyo Bunrika Daikagu (A) 4 (1951), 177-194.

[9] M. Schaps, 'Deformations of finite dimensional algebras and their idempotents,' Trans. Amer. Math. Soc. 307 (1988), 843-856.

[10] M. Schaps, 'A modular version of Maschke's theorem for group algebras of finite representation type and for blocks with cyclic defect group' preprint, Bar-Ilan University.

[11] D. Wallace, 'On the radical of a group algebra,' Proc. Amer. Math. Soc. 12 (1961), 133-137.

Bar-Ilan University

52100 Ramat-Gan

Israel 